$$
\begin{array}{ll}
\text { Research Square } & \begin{array}{l}
\text { Preprints are preliminary reports that have not undergone peer review. } \\
\text { They should not be considered conclusive, used to inform clinical practice, } \\
\text { or referenced by the media as validated information. }
\end{array}
\end{array}
$$

\title{
The Clinicopathological Features of BRG1-deficient Non-small Cell Lung Cancer and Its Response to Immunotherapy: A Single-center Retrospective Study
}

Xiaoyan Chen ( $\nabla$ cxy11832@rjh.com.cn)

Shanghai Jiao Tong University Medical School Affiliated Ruijin Hospital https://orcid.org/0000-0001-5665-3586

Jing Zhang

Tongji University Affilliated East Hospital: Tongji University Dongfang Hospital

Runze Zhao

Shanghai Jiao Tong University Medical School Affiliated Ruijin Hospital

Haimin Xu

Shanghai Jiao Tong University Medical School Affiliated Ruijin Hospital

Lei Dong

Shanghai Jiao Tong University Medical School Affiliated Ruijin Hospital

\section{Research Article}

Keywords: BRG1-deficient NSCLC, clinicopathological features, immune response

Posted Date: August 24th, 2021

DOI: https://doi.org/10.21203/rs.3.rs-830549/v1

License: (c) (i) This work is licensed under a Creative Commons Attribution 4.0 International License. Read Full License 


\section{Abstract}

Purpose

BRG1-deficient NSCLCs have been more intriguing recently for its highly aggressive clinical behavior and no effective therapies. This study characterized the clinical and pathological features of BRG1-deficient NSCLCs and investigated their response to immunotherapy.

Methods

Forty-seven cases with BRG1-deficient NSCLC were included. Immunohistochemical markers such as CK7, TTF-1, NapsinA, P40, HepPar-1, Ki-67, BRM, ARID1A and ARID1B were stained. Meanwhile, the PD-L1 expression level, overall survival, progression-free survival and disease control rate of patients received immunotherapy were evaluated.

Results

This study revealed that: (1) Patients with BRG1-deficient NSCLC have a male predominance(89.4\%), smoker enrichment(76.6\%) and poor prognosis(median OS: 7.0 months for advanced stage). (2) Histologically, BRG1-deficient NSCLCs presented significant morphological diversity and no lepidic pattern. Inflammatory infiltration and tumor necrosis was a prominent feature. Immunohistochemical analyses showed a distinctive uniform immunophenotype (TTF$1-/ \mathrm{NapsinA}-/ \mathrm{CK} 7+)$ in $60.9 \%$ (28/46) of cases and HepPar-1 positive in $46.5 \%$ (20/43) of cases. BRM loss or significant reduction coexisted in $11.8 \%$ (4/34) of cases. No case (0/37) showed loss of ARID1A or ARID1B. (3) For twenty-nine patients with advanced stage, eight patients had received immunotherapy and 4 cases achieved a sustainable clinical response with the disease control rate of $50 \%$. ICls treated patients had better OS than those who received non-ICls treatment settings (median OS, $27.0 \mathrm{~m}$ versus $6.0 \mathrm{~m}, \mathrm{p}=0.02$ ). Moreover, patients received ICls have a median PFS of 17.0 months, while, median PFS on platinum doublet chemotherapy were only 6.0 months $(p=0.04)$.

Conclusion

BRG1-deficient NSCLC showed diverse histopathological patterns and a unique immunohistochemical phenotype. ICls-based immunotherapy was a beneficial therapy for BRG1- deficient NSCLC.

\section{Introduction}

Mammalian switch/sucrose non-fermentable (mSWI/SNF) chromatin remodeling complex is a group of polyprotein complexes that hydrolyze ATP to break up the DNA-histone interaction, mobilize nucleosome, and subsequently change the structure of chromatin in a non-covalent way. This complex and its subunits participate in tissue genesis and differentiation through multiple cell signaling processes by altering transcription, duplication, repair and reconstruction of chromatin. The mutation rate of $\mathrm{mSWI} / \mathrm{SNF}$ subunits has been up to $20 \%$ in all human cancers, and their tumor-suppressive role has been established by early studies (Versteege et al.1998; Wilson.2011; Masliah-Planchon et al.2015). SMARCA4, the essential ATPase subunit of mSWI/SNF complex, is located on chromosome 19p. It encodes protein BRG1, which interacts with multiple cell cycle regulatory proteins including Rb, p53 etc. Thus, loss of SMARCA4 subunit causes cell cycle defects and the following tumorigenesis (Alessio et al. 2010). Furthermore, the dysfunctional BRG1 upregulates the transcription of several key tumor-associated genes, which also contributes to tumorigenesis(Orvis et al.2014). Both gene mutations and protein deficiency in several subunits of mSWI/SNF (SMARCA4/BRG1, SMARCA2/BRM, ARID1A and ARIDIB) have been identified in a variable proportion of non-small cell lung cancer (NSCLC) (Huang et al.2015; Jones, Siân et al.2012). Previous studies have shown that SMARCA4/BRG1-deficiency presented in about 30\% of NSCLC cell lines and 10$15 \%$ of clinical tissue samples (Girard et al. 2000; Reisman et al.2003; Oike et.al.2013). In recent years, BRG1-deficient non-small cell lung cancer (NSCLC) has been gradually classified as a special subtype of NSCLCs. However, most of these studies focused on NSCLC cell lines, while detailed clinicopathological and morphological studies on BRG1-deficient tumors are lacking.

The prognosis for patients with BRG1-deficient NSCLC is poor because therapeutic strategies for this phenotype of NSCLC have not been established. Existing evidences suggested BRG1-deficient NSCLC was incompatible with classic driver genes such as EGFR, ALK, ROS1, which may lead to its insensitivity to traditional targeted drugs (Araujo et al.2015). A previous study reported that low expression of SMARCA4/BRG1 was a novel predictive biomarker for increasing sensitivity to platinum-based chemotherapy in NSCLC (Bell et al.2016). However, this is challenged by another study, in which the median progression-free survival for patients with BRG1-deficient NSCLC was only 38 days after first-line platinum doublet chemotherapy (Dagogo-Jack et al.2020). In addition, several studies have implicated that inactivation of the mSWI/SNF complex had enhanced tumor sensitivity to immune checkpoint inhibitors(ICls) (Henon et al.2019; Li et al. 2019; Miao et al.2018). However, little is known about the response of BRG1-deficient NSCLCs to immunotherapy.

Herein, we described the clinical, pathomorphological and immunohistochemical characteristics of 47 NSCLC cases with total loss, partial loss or significant reduction of BRG1 protein (collectively referred to as BRG1-deficient) in detail. In addition, we evaluated the PD-L1 expression level in BRG1-deficient NSCLC.

For patients receiving immunotherapy, we collected clinical and imaging information of these patients. The results can provide more references for evaluating the response of BRG1-deficient NSCLCs to immunotherapy.

\section{Material And Methods}

2.1 Data collection and Statistical analysis

Forty-seven cases of BRG1-deficient NSCLC (including biopsy and surgical cases) were diagnosed during daily practice in the Department of Pathology of Shanghai Ruijin Hospital from October 1st 2015 to May 31th 2020. The study was conducted under the approval by the Institutional Review Board. Ethical 
approval was granted by the clinical Research Ethics Committee of Shanghai Ruijin Hospital, Jiaotong University. Signed informed consents were obtained from all patients for the acquisition and use of tissue samples and anonymized clinical information. All cases were confirmed by repeating the BRG1 immunohistochemistry staining with whole sections. In addition, these tumors were stained for CK7, TTF-1, NapsinA, P40, HepPar-1, Ki-67, PD-L1(22C3), BRM, ARID1A and ARID1B. Other markers such as CK-pan, CD34, Claudin 4, SALL4, SOX2, CgA, SYN, CD56 and PAX-8 were stained to exclude thoracic BRG1deficient undifferentiated tumors and metastatic tumors. All sections were reviewed and evaluated by two pulmonary pathologists. The information about antibodies used in this study are shown in Supplemental Table 1.

The patients' medical records including gender, age, smoking history, clinical stage and pathological morphology were reviewed and summarized. The 8th edition of the TNM classification for lung cancer (International Association for the Study of Lung Cancer) was used for tumor staging. We acquired follow-up data from outpatient visits or by telephone. Overall survival (OS) was defined as the days from pathological diagnosis to death or July 31th, 2021. For patients receiving immunotherapy, progression-free survival (PFS) was defined as the days from the start of immunotherapy to disease progression detected by imaging or death, and disease control rate(DCR) was defined as the proportion of patients who had a best response rating of complete response, partial response or stable disease. The cut off date for this analysis was July 31th, 2021. The level of significance was defined as $p<0.05$. Median OS and PFS was calculated using the Kaplan-Meier analysis. All statistical analyses were performed using GraphPad Software, Inc.(version 6.0). Categorical variables were presented as percentages.

\subsection{Immunohistochemical and genetic evaluation}

The specimens were fixed with $10 \%$ formalin and embedded in paraffin. Serial 4- $\mu$ m-thick sections were prepared and used for hematoxylin and eosin (H\&E) staining and immunohistochemistry (IHC). IHC staining for PD-L1(22C3) was performed on the Dako Autostainer Link 48 platform and for other antigens was performed on an automated immunohistochemistry system (Bond-III; Leica Biosystems, Buffalo Grove, Illinois, USA) using BOND Polymer Refine DS9800 Detection kit according to the manufacture's specifications. Total loss (TL) of BRG1 was defined as complete absence of nuclear expression in tumor cells compared with strong nuclear expression in the background stromal cells and/or non-neoplastic epithelial cells. Partial loss(PL) of BRG1 was defined as expression present in some tumor cell nuclei but absent in other tumor cell nuclei, while with strong staining in surrounding normal epithelial cells, mesenchymal cells and inflammation cells. "Significant reduction (SR)" of BRG1 was defined as uniformly-attenuated expression in tumor cell nuclear staining compared with surrounding normal epithelial cells, mesenchymal cells and inflammatory cells. For PD-L1, the proportion of viable tumor cells exhibiting complete or partial membranous staining (at least $1+$ intensity) was evaluated. PD-L1 scores were categorized as $<1 \%$ (negative) or $\geq 1 \%$ tumor cells, and $<50 \%$ (low) or $\geq 50 \%$ tumor cells(high) (Hirsch et al.2017). For several cases, clinically-relevant molecular testing for EGFR mutations, KRAS mutations, gene rearrangement of ALK/EML4 and ROS1 has been performed routinely and data were summarized in this study.

\subsection{Patient and public involvement statement}

No patients or public were involved in setting the research question or the outcome measures, nor were they involved in the design and implementation of the study. There are no plans to involve patients in dissemination.

\section{Results}

\subsection{Clinical features of BRG1-deficient NSCLCs}

The main clinical features of the 47 cases with BRG1-deficient (including total loss, partial loss and significant reduction of BRG1) NSCLC confirmed by immunohistochemistry are summarized in Table 1. This study included $42(89.4 \%)$ male and $5(10.6 \%)$ female patients, whose ages ranged from 37 to 83 years with the mean age of 63.0 years old. Thirty-six (76.4\%) patients had smoking history. Eighteen patients underwent surgical resection of the primary tumor: nine(50.0\%) of them received preoperative or postoperative adjuvant chemotherapy and five (27.8\%) patients suffered recurrence within the first year after surgery. The other patients $(n=29)$ were in the advanced stage or can not tolerate surgery. Among twenty-seven patients whose therapeutic information were available, eleven(37.9\%) patients received platinum doublet chemotherapy, eight(27.6\%) patients were treated with ICls-based immunotherapy. Four(13.8\%) patients received chemotherapy combined other therapies include anti-angiogenesis, radiotherapy and Gamma Knife and the other four(13.8\%) patients received palliative treatment $(n=2)$, tyrosine kinase inhibitor $(T K I)(n=1)$, and Gamma Knife $(n=1)$. Up to July 31th, 2021, thirteen patients $(27.7 \%)$ stay alive with disease free after complete tumor resection, five patients(10.6\%) still live with tumor and twenty-nine(61.7\%) patients had died. Meanwhile, for twenty-nine patients who with advanced stage, $82.8 \%(24 / 29)$ patients died before the end of this study, with the median OS of 7.0 months.

\subsection{The efficacy of immunotherapy of BRG1-deficent NSCLC}

Eight patients received anti-PD-1/PD-L1 inhibitors treatment(Table 2). Among them, patient No.19 disease progression after two cycles of cisplatin, pemetrexed and avastin as the first-line treatment, and then was treated with pembrolizumab combined with docetaxel as the second-line treatment. Patient No.30 received sintilimab as the second-line treatment after the first-line treatment (cisplatin and pemetrexed) failed. Patient No.35 received pembrolizumab combined with anlotinib as the third-line treatment. The CT scans demonstrated a sustained partial response after 21 cycles of intravenous administration of pembrolizumab (Fig 1A-1C). Until July 31, 2021, four patients mentioned above continue to benefit from immunotherapy and their PFS were not reached (exceeding 37, 20, 14 and 31 months, respectively). Three patients (No.20, No.37 and No.43) showed no response to immunotherapy alone or combined with chemotherapy, and their PFS were 5, 3 and 2 months respectively. Patient No.44 who benefited from nivolumab combined with chemotherapy as the first-line treatment for 14 months but died of severe myelosuppression. Overall, for eight patients who received immunotherapy, 4 cases achieved a sustainable clinical benefit with the DCR of $50 \%$. Furthermore, for patients who with advanced BRG1-deficient NSCLC, ICls ever treated patients $(\mathrm{n}=8)$ had better median OS than those who received non-ICls treatment settings $(n=19)$ including chemotherapy, anti-angiogenesis therapy, TKI, radiotherapy, Gamma Knife and palliative 
therapy (median OS, $27.0 \mathrm{~m}$ versus $6.0 \mathrm{~m}, \mathrm{p}=0.02$ ). Moreover, patients received ICls have a median PFS of 17.0 months, while median PFS on platinum doublet chemotherapy $(n=11)$ were only 6.0 months $(p=0.04)$ (Fig 2A-2B).

\subsection{Pathological, immunohistochemical and molecular findings in BRG1-deficient NSCLCs}

The histological specimens were obtained from lobectomy resection $(n=18)$ or needle biopsies from primary lesions and metastatic lesions $(n=29)$. The histopathological profiles of 47 cases were: 42 adenocarcinomas, 1 non-keratinizing squamous cell carcinoma, 1 adenosquamous carcinoma, 2 non-small cell carcinomas, not otherwise specified, and 1 pleomorphic carcinoma (Table 3-1 and Table 3-2).Among the 42 adenocarcinomas, 31 were pure poorlydifferentiated solid adenocarcinoma with minimal abortive small lumina and scattered goblet-like cells (Fig.3A-3B), 6 were solid adenocarcinoma with a small amount of acinar-like component (additional foci of micropapillary component seen in one of them) , 2 were papillary adenocarcinoma with a small amount of solid and acinar-like components, 2 were pure acinar adenocarcinoma and 1 was mucinous adenocarcinoma. No lepidic growth pattern was seen in all adenocarcinomas. Most of the tumors were composed of large polygonal tumor cells arranged in compact solid nests and sheets (Fig.3A, Fig.3C) except for differentiated acinar-like, papillary and micropapillary areas. Tumor cells frequently showed clear cytoplasm, vesicular chromatin(Fig.3C-3D), distinct eosinophilic nucleoli and a high mitotic rate (13 mitoses per $2 \mathrm{~mm}^{2}$ ) (Fig.3E). Scattered pleomorphic giant cells and bizzare cells were seen in 5 cases(Fig.3B), and 1 case with extensive giant cells was diagnosed as pleomorphic carcinoma. Of the 47 cases, 32 cases(68.1\%) showed more or less rhabdoid tumor cells including 2 cases with frankly rhabdoid tumor cells(Fig.3E) , 37 cases (78.7\%) showed cytoplasm hyaline secretory globules of varying size (Fig.3F), 37 cases(78.7\%) showed necrosis(Fig.3A). Extensive interstitial and intraepithelial infiltrate of plasma cells, lymphocytes and neutrophils was a frequent finding. (Fig.3B-3C,3E).

In IHC staining, total loss (Fig.4A-4F), partial loss (Fig.4G) and significant reduction(Fig.4H), of BRG1 were seen in 36, 6, and 5 cases respectively. Of the 42 adenocarcinomas, TTF-1 was negative in 27 cases (64.3\%), strong and diffuse positive in 7 cases, and partially or weakly positive in 8 cases. Of note, in 4 of 6 BRG-1 partial loss cases, TTF-1 was positive only in the subpopulation of tumor cells with BRG1 intact staining. Of the 41 adenocarcinomas available for NapsinA staining, 31 (75.6\%) were negative, 3 were strong and diffuse positive,7 were partially positive. P40 was strongly and diffusely positive in the only squamous cell carcinoma. In one adenosquamous carcinoma and one pleomorphic carcinoma, P40 was positive in $20 \%$ tumor cells. Cytokeratin 7 was expressed in 93.6\% (44/47) NSCLCs and 92.9\% (39/42) adenocarcinomas(Fig.4I), but not expressed in 3 solid adenocarcinomas. HepPar-1 showed strong granular cytoplasmic expression in 46.5\% (20/43) NSCLCs and $48.7 \%$ (19/39) adenocarcinomas(Fig.4J). Ki-67 index ranged from $20 \%$ to $90 \%$ with a mean of $60 \%$. Of the 34 cases available for BRM staining, 2 showed total loss (Fig.4K), 1 showed significant reduction (Fig.4L) and 1 showed partially reduction. No case showed ARID1A or ARID1B loss in the 37 cases detected. PD-L1 expression was detected in 44 BRG1-deficient NSCLCs with adequate tissue samples, of which 20 (45.5\%) showed positive expression, including 9 (20.5\%) with high expression and $11(25 \%)$ with low expression. Among 25 tumors with genetic information available, 2 (8.0\%) had EGFR mutation and 5 (20.0\%) had KRAS mutation. Other genetic alterations including the rearrangement of ALK/EML4 and ROS1 were not identified.

\section{Discussion}

BRG1-deficient NSCLCs have been more intriguing recently for its highly aggressive clinical behavior and no effective therapies. We summarized the clinical, pathomorphological and immunohistochemical characteristics of BRG1-deficient NSCLCs in detail. Patients with BRG1-deficient NSCLCs tend to be male with tobacco exposure, as a previous study reported (Decroix et al. 2020). About $27.8 \%$ of patients suffered recurrence in the first year after lung surgery. For twenty-nine patients who were in the advanced stage, $82.8 \%(24 / 29)$ patients died before the end of this study, with the median 0 S of only 7.0 months.

SMARCA4 mutation usually coexists with mutation of KRAS, LKB1, KEAP1, P16 and P53 genes, which suggests that BRG1-deficient NSCLC may be insensitive to traditional treatments such as chemotherapy, radiotherapy and targeted inhibitors (Dagogo-Jack et al.2020). The ICls-based immunotherapy has achieved remarkable success in advanced NSCLCs. Existing evidences revealed that when compared to wild-type subgroups, the inactiviation of SMARCA4/BRG1 may predict poorer clinical response to ICls (Zhou et al.2020). However, little is known about the ICls efficacy of the BRG1-deficient NSCLC when compared to the traditional therapeutic settings. In our cohort, for advanced BRG1-deficient NSCLCs, ICls ever treated patients had better OS than those who received non-ICls treatment settings including chemotherapy, anti-angiogenesis therapy, TKI, radiotherapy, Gamma Knife and palliative therapy (median OS, $27.0 \mathrm{~m}$ versus $6.0 \mathrm{~m}, \mathrm{p}=0.02$ ). Moreover, patients received ICls have an improved median PFS of 17.0 months, while median PFS on platinum doublet chemotherapy were only 6.0 months $(p=0.04)$. Notably, three patients benefited from ICls therapy as second or third-line treatment even after failing from traditional settings. These data suggest that patients with BRG1-deficient NSCLC may be more sensitive to immunotherapy than traditional settings such as chemotherapy. However, as a retrospective study, the number, age, stage and the treatment lines among the groups have unbalanced differences, without setting the control or matching the patients, which may affect the conclusion. Therefore, randomized controlled trials are necessity to examine efficacy difference between IClsbased immunotherapy and traditional therapies for BRG1-deficient NSCLCs.

The PD-L1 expression level and tumor mutation burden(TMB) were important factors for predicting ICls response (Carbone et al. 2017; Rizvi et al. 2015). Recently, a case report indicated that BRG1-deficient NSCLC had higher level of PD-L1 and TMB, and therefore could generate sustained response to nivolumab, the inhibitor of PD-L1(Naito et al. 2019). However, two cases in our cohort showed sustainable response to ICls even with low PD-L1 expression level. Furthermore, another study indicated that NSCLC with SMARCA4 mutation harbored high TMB but infrequently(15\%) had high PD-L1(Dagogo-Jack et al.2020)(13). Therefore, despite the expression level of PD-L1, other effective predictors such as TMB may also account for the response of BRG1-deficient NSCLCs to immunotherapy.

In our study, BRG1-deficient NSCLCs showed varied histological types, including adenocarcinoma, squamous-cell carcinoma, adenosquamous carcinoma and pleomorphic carcinoma, as early studies had reported (Agaimy et al.2017; Naito et al.2019; Nambirajan et al. 2021; Herpel et al. 2017). Consistent with previous results, no lepidic pattern was found in our study (Matsubara et al.2013). Notably, a smaller area of micro-papillary pattern was observed in one solid 
predominant adenocarcinoma, which has not been reported previously. Significant morphological diversity makes it difficult to predict BRG1 deficiency only by histomorphometric analysis. Accordingly, we consider it necessary to detect BRG1 staining in all NSCLCs. Tumor cells of BRG1-deficient NSCLCs showed overt cytological atypia even in papillary or acinus predominant adenocarcinoma. More or less cytoplasmic hyaline droplets (their nature remains unknown) can be seen in most cases. Multifocal and irregular tumor necrosis accompanied by inflammatory infiltration were the prominent features. Frequent necrosis, overt cytological atypia, high mitotic rate and high proliferation index all predicted the aggressive behavior of BRG1-deficient NSCLCs. The inflammatory cells were seen in both interstitium and tumor nests, suggesting inflammation may play an important role in the establishment of tumor microenvironment.

Partial loss and significant reduction of BRG1 in NSCLCs have only been occasionally described in literatures (Dagogo-Jack et al.2020; Nambirajan et al. 2021; Herpel et al. 2017). Partial loss of BRG1 were frequently seen in our study possibly due to the whole-section immunohistochemistry staining rather than tissue microarray. There was a relatively clear boundary between the BRG1-deficient and BRG1-intact areas in the 6 cases with partial loss. Among them, 5 showed no significant morphological difference between the two areas while one showed loss of BRG1 in solid growth pattern but intact BRG1 in acinus pattern. One study presumed that loss of BRG1 occurs at the early phase of carcinogenesis of lung adenocarcinoma (Matsubara et al.2013). However, findings in our cases with partial loss of BRG1 suggested that this presumption needs to be reconsidered. BRG1-deficient lung adenocarcinomas were typically negative for TTF-1 and positive for CK7 and HepPar-1(Hirsch et al.2017; Nambirajan et al.2020). In our study, TTF-1 and NapsinA was negative in 64.3\% and 75.6\% BRG1deficient adenocarcinomas respectively. Interestingly, in BRG1-partially lost cases, 4 were positive for TTF-1 and 3 were positive for NapsinA only in the area with BRG1 expression intact. CK7 was positive in $92.9 \%$ of adenocarcinomas and $93.6 \%$ of all NSCLCs, which was consistent with previous studies. HepPar-1 was reported to be positive in 85\% BRG1-deficient lung adenocarcinomas by Agaimy et al(2017). However, in our study, HepPar-1 was only positive in $48.7 \%$ of adenocarcinomas and $46.5 \%$ of all NSCLCs. HepPar-1 staining showed strong granular cytoplasmic(mitochondrial) pattern as seen in normal liver and hepatoid and hepatocellular carcinomas. Therefore, when HepPar-1 is positive in biopsy sample, it is necessary to search for BRG1-deficient lung cancer, hepatocellular carcinoma and hepatoid carcinoma. As a further test, we examined the expression of other three subunits of the SWI/SNF complex in BRG1deficient NSCLCs. Four cases (11.8\%) had loss or reduction of BRM and no case showed ARID1A or ARID1B loss. Interestingly, in one case with partial loss of BRG1 (No.41), the BRM expression decreased exactly in BRG1 loss area, which further suggested the underlying relationship between BRG1 and BRM. In a study, ARID1A, ARID1B, BRG1 and BRM were lost in $1.3 \%, 0.3 \% 2.4 \%$ and $2.4 \%$ of NSCLCs respectively, suggesting that loss of ARID1A or ARID1B was not common, especially for ARID1B (Naito et al.2019).

In our cohort, there was minimal overlap between BRG1 deficiency and actionable oncogenic driver alterations. KRAS mutations rather than EGFR mutations occurred frequently in BRG1-deficient NSCLC. As we all known that, KRAS gene mutations are the most prevalent molecular alteration detected in human lung adenocarcinoma, accounting for up to $25 \%$ of all genetic mutations. The prior efforts to develop specific targeted inhibitors for the KRAS-mutant patients has been unsuccessful (Ferrer et al.2018). A recent study had revealed that SMARCA4 mutation was a risk factor for KRAS-mutant patients with lung adenocarcinoma (Liu et al.2021). On the other hand, IHC for BRG1 can capture SMARCA4-deficient tumor which is associated with SMARCA4 mutations (Dagogo-Jack et al.2020). Therefore, evaluation of BRG1 expression by IHC may further of KRAS-mutant patients with lung adenocarcinoma.

\section{Conclusion}

In summary, our study demonstrated that BRG1-deficient NSCLC is enriched in smokers, predominantly occurs in male and has aggressive clinical behavior. Given the varied histological types and significant morphological diversity in BRG1-deficient NSCLC, we recommend staining BRG1 in all NSCLCs in clinical practice. Importantly, ICls-based immunotherapy may be a promising therapy for BRG1-deficient NSCLCs, underscoring the necessity of developing multicenter clinical trials to confirm the benefits of immunotherapy in this subtype of NSCLC.

\section{Declarations}

Ethics approval: The study was conducted under the approval by the Institutional Review Board. Ethical approval was granted by the clinical Research Ethics Committee of Shanghai Ruijin Hospital, Jiaotong University (number:202005).

Patient consent for publication: All patients signed consent for publication.

Authors' contributions: Conceptualization, Xiaoyan Chen; Methodology, Haimin Xu; Validation, Xiaoyan Chen and Jing Zhang; Formal Analysis, Xiaoyan Chen; Investigation, Jing Zhang; Resources \& Data Curation, Lei Dong; Writing - Review \& Editing, Jing Zhang and Runze Zhao; Supervision, Xiaoyan Chen;

Data availability statement: No additional data available.

Funding: Not applicable.

Disclosure: The authors declared that they have no conflict of interest.

Acknowledgements: Not applicable.

\section{References}

1. Versteege, I., Sévenet, N., Lange, J., Rousseau-Merck, M. F., Ambros, P., Handgretinger, R., Aurias, A., \& Delattre, O. (1998). Truncating mutations of hSNF5/INI1 in aggressive paediatric cancer. Nature, 394(6689), 203-206.

2. Wilson, B. G., \& Roberts, C. W. (2011). SWI/SNF nucleosome remodellers and cancer. Nature reviews. Cancer, 11(7), $481-492$. 
3. Masliah-Planchon, J., Bièche, I., Guinebretière, J. M., Bourdeaut, F., \& Delattre, O. (2015). SWI/SNF chromatin remodeling and human malignancies. Annual review of pathology, 10, 145-171. doi:10.1146/annurev-pathol-012414-040445

4. Alessio, N., Squillaro, T., Cipollaro, M., Bagella, L., Giordano, A., \& Galderisi, U. (2010). The BRG1 ATPase of chromatin remodeling complexes is involved in modulation of mesenchymal stem cell senescence through RB-P53 pathways. Oncogene, 29(40), 5452-5463. doi:10.1038/onc.2010.285

5. Orvis, T., Hepperla, A., Walter, V., Song, S., Simon, J., Parker, J., Wilkerson, M. D., Desai, N., Major, M. B., Hayes, D. N., Davis, I. J., \& Weissman, B. (2014). BRG1/SMARCA4 inactivation promotes non-small cell lung cancer aggressiveness by altering chromatin organization. Cancer research, 74(22), 64866498. doi:10.1158/0008-5472

6. Huang, H. T., Chen, S. M., Pan, L. B., Yao, J., \& Ma, H. T. (2015). Loss of function of SWI/SNF chromatin remodeling genes leads to genome instability of human lung cancer. Oncology reports, 33(1), 283-291. doi:10.3892/or.2014.3584

7. Jones, S., Li, M., Parsons, D. W., Zhang, X., Wesseling, J., Kristel, P., Schmidt, M. K., Markowitz, S., Yan, H., Bigner, D., Hruban, R. H., Eshleman, J. R., lacobuzio-Donahue, C. A., Goggins, M., Maitra, A., Malek, S. N., Powell, S., Vogelstein, B., Kinzler, K. W., Velculescu, V. E., ... Papadopoulos, N. (2012). Somatic mutations in the chromatin remodeling gene ARID1A occur in several tumor types. Human mutation, 33(1), 100-103. doi:10.1002/humu.21633

8. L Girard , S Zöchbauer-Müller, A K Virmani, et al. Genome-wide allelotyping of lung cancer identifies new regions of allelic loss, differences between small cell lung cancer and non-small cell lung cancer, and loci clustering. Cancer Res. 2000 Sep 1;60(17):4894-4906.

9. Reisman DN, Sciarrotta J, Wang W, Funkhouser WK, Weissman BE. Loss of BRG1/BRM in human lung cancer cell lines and primary lung cancers: correlation with poor prognosis. Cancer Res. 2003 Feb 1;63(3):560-566.

10. Oike, T., Ogiwara, H., Tominaga, Y., Ito, K., Ando, O., Tsuta, K., Mizukami, T., Shimada, Y., Isomura, H., Komachi, M., Furuta, K., Watanabe, S., Nakano, T., Yokota, J., \& Kohno, T. (2013). A synthetic lethality-based strategy to treat cancers harboring a genetic deficiency in the chromatin remodeling factor BRG1. Cancer research, 73(17), 5508-5518. doi:10.1158/0008-5472.CAN-12-4593

11. Araujo, L. H., Timmers, C., Bell, E. H., Shilo, K., Lammers, P. E., Zhao, W., Natarajan, T. G., Miller, C. J., Zhang, J., Yilmaz, A. S., Liu, T., Coombes, K., Amann, J., \& Carbone, D. P. (2015). Genomic Characterization of Non-Small-Cell Lung Cancer in African Americans by Targeted Massively Parallel Sequencing. Journal of clinical oncology: official journal of the American Society of Clinical Oncology, 33(17), 1966-1973. doi:10.1200/JC0.2014.59.2444

12. Bell, E. H., Chakraborty, A. R., Mo, X., Liu, Z., Shilo, K., Kirste, S., Stegmaier, P., McNulty, M., Karachaliou, N., Rosell, R., Bepler, G., Carbone, D. P., \& Chakravarti, A. (2016). SMARCA4/BRG1 Is a Novel Prognostic Biomarker Predictive of Cisplatin-Based Chemotherapy Outcomes in Resected Non-Small Cell Lung Cancer. Clinical cancer research: an official journal of the American Association for Cancer Research, 22(10), 2396-2404. doi:10.1158/1078-0432.CCR15-1468

13. Dagogo-Jack, I., Schrock, A. B., Kem, M., Jessop, N., Lee, J., Ali, S. M., Ross, J. S., Lennerz, J. K., Shaw, A. T., \& Mino-Kenudson, M. (2020). Clinicopathologic Characteristics of BRG1-Deficient NSCLC. Journal of thoracic oncology: official publication of the International Association for the Study of Lung Cancer, 15(5), 766-776. doi:10.1016/j.jtho.2020.01.002

14. Henon, C., Blay, J. Y., Massard, C., Mir, O., Bahleda, R., Dumont, S., Postel-Vinay, S., Adam, J., Soria, J. C., \& Le Cesne, A. (2019). Long lasting major response to pembrolizumab in a thoracic malignant rhabdoid-like SMARCA4-deficient tumor. Annals of oncology: official journal of the European Society for Medical Oncology, 30(8), 1401-1403. doi:10.1093/annonc/mdz160

15. Li, L., Li, M., Jiang, Z., \& Wang, X. (2019). ARID1A Mutations Are Associated with Increased Immune Activity in Gastrointestinal Cancer. Cells, 8(7), 678. doi: $10.3390 /$ cells 8070678

16. Miao, D., Margolis, C. A., Gao, W., Voss, M. H., Li, W., Martini, D. J., Norton, C., Bossé, D., Wankowicz, S. M., Cullen, D., Horak, C., Wind-Rotolo, M., Tracy, A., Giannakis, M., Hodi, F. S., Drake, C. G., Ball, M. W., Allaf, M. E., Snyder, A., Hellmann, M. D., ... Van Allen, E. M. (2018). Genomic correlates of response to immune checkpoint therapies in clear cell renal cell carcinoma. Science (New York, N.Y.), 359(6377), 801-806. doi:10.1126/science.aan5951

17. Hirsch, F. R., McElhinny, A., Stanforth, D., Ranger-Moore, J., Jansson, M., Kulangara, K., Richardson, W., Towne, P., Hanks, D., Vennapusa, B., Mistry, A., Kalamegham, R., Averbuch, S., Novotny, J., Rubin, E., Emancipator, K., McCaffery, I., Williams, J. A., Walker, J., Longshore, J., ... Kerr, K. M. (2017). PD-L1 Immunohistochemistry Assays for Lung Cancer: Results from Phase 1 of the Blueprint PD-L1 IHC Assay Comparison Project. Journal of thoracic oncology: official publication of the International Association for the Study of Lung Cancer, 12(2), 208-222. doi:10.1016/j.jtho.2016.11.2228

18. Decroix, E., Leroy, K., Wislez, M., Fournel, L., Alifano, M., Damotte, D., \& Mansuet-Lupo, A. (2020). Les tumeurs thoraciques SMARCA4 déficientes: une nouvelle entité [SMARCA4-deficient thoracic tumors: A new entity]. Bulletin du cancer, 107(1), 41-47. doi:10.1016/j.bulcan.2019.12.001

19. Zhou, H., Shen, J., Liu, J., Fang, W., \& Zhang, L. (2020). Efficacy of Immune Checkpoint Inhibitors in SMARCA4-Mutant NSCLC. Journal of thoracic oncology: official publication of the International Association for the Study of Lung Cancer, 15(8), e133-e136. doi:10.1016/j.jtho.2020.03.030

20. Carbone, D. P., Reck, M., Paz-Ares, L., Creelan, B., Horn, L., Steins, M., Felip, E., van den Heuvel, M. M., Ciuleanu, T. E., Badin, F., Ready, N., Hiltermann, T., Nair, S., Juergens, R., Peters, S., Minenza, E., Wrangle, J. M., Rodriguez-Abreu, D., Borghaei, H., Blumenschein, G. R., Jr, ... CheckMate 026 Investigators (2017). First-Line Nivolumab in Stage IV or Recurrent Non-Small-Cell Lung Cancer. The New England journal of medicine, 376(25), 24152426. doi:10.1056/NEJMoa1613493

21. Rizvi, N. A., Hellmann, M. D., Snyder, A., Kvistborg, P., Makarov, V., Havel, J. J., Lee, W., Yuan, J., Wong, P., Ho, T. S., Miller, M. L., Rekhtman, N., Moreira, A. L., Ibrahim, F., Bruggeman, C., Gasmi, B., Zappasodi, R., Maeda, Y., Sander, C., Garon, E. B., ... Chan, T. A. (2015). Cancer immunology. Mutational landscape determines sensitivity to PD-1 blockade in non-small cell lung cancer. Science (New York, N.Y.), 348(6230), 124-128. doi:10.1126/science.aaa1348

22. Naito, T., Umemura, S., Nakamura, H., Zenke, Y., Udagawa, H., Kirita, K., Matsumoto, S., Yoh, K., Niho, S., Motoi, N., Aokage, K., Tsuboi, M., Ishii, G., \& Goto, K. (2019). Successful treatment with nivolumab for SMARCA4-deficient non-small cell lung carcinoma with a high tumor mutation burden: A case report. Thoracic cancer, 10(5), 1285-1288. doi:10.1111/1759-7714.13070

23. Agaimy, A., Fuchs, F., Moskalev, E. A., Sirbu, H., Hartmann, A., \& Haller, F. (2017). SMARCA4-deficient pulmonary adenocarcinoma: clinicopathological, immunohistochemical, and molecular characteristics of a novel aggressive neoplasm with a consistent TTF1neg/CK7pos/HepPar-1pos 
immunophenotype. Virchows Archiv: an international journal of pathology, 471(5), 599-609. doi:10.1007/s00428-017-2148-5

24. Naito, T., Udagawa, H., Umemura, S., Sakai, T., Zenke, Y., Kirita, K., Matsumoto, S., Yoh, K., Niho, S., Tsuboi, M., Ishii, G., \& Goto, K. (2019). Non-small cell lung cancer with loss of expression of the SWI/SNF complex is associated with aggressive clinicopathological features, PD-L1-positive status, and high tumor mutation burden. Lung cancer (Amsterdam, Netherlands), 138, 35-42. doi:10.1016/j.lungcan.2019.10.009

25. Nambirajan, A., Singh, V., Bhardwaj, N., Mittal, S., Kumar, S., \& Jain, D. (2020). SMARCA4/BRG1-Deficient Non-Small Cell Lung Carcinomas: A Case Series and Review of the Literature. Archives of pathology \& laboratory medicine, 10.5858/arpa.2019-0633-0A. doi:10.5858/arpa.2019-0633-0A

26. Herpel, E., Rieker, R. J., Dienemann, H., Muley, T., Meister, M., Hartmann, A., Warth, A., \& Agaimy, A. (2017). SMARCA4 and SMARCA2 deficiency in nonsmall cell lung cancer: immunohistochemical survey of 316 consecutive specimens. Annals of diagnostic pathology, 26, 4751. doi:10.1016/j.anndiagpath.2016.10.006

27. Matsubara, D., Kishaba, Y., Ishikawa, S., Sakatani, T., Oguni, S., Tamura, T., Hoshino, H., Sugiyama, Y., Endo, S., Murakami, Y., Aburatani, H., Fukayama, M., \& Niki, T. (2013). Lung cancer with loss of BRG1/BRM, shows epithelial mesenchymal transition phenotype and distinct histologic and genetic features. Cancer science, 104(2), 266-273. doi:10.1111/cas.12065

28. Ferrer, I., Zugazagoitia, J., Herbertz, S., John, W., Paz-Ares, L., \& Schmid-Bindert, G. (2018). KRAS-Mutant non-small cell lung cancer: From biology to therapy. Lung cancer (Amsterdam, Netherlands), 124, 53-64. doi:10.1016/j.lungcan.2018.07.013

29. Liu, L., Ahmed, T., Petty, W. J., Grant, S., Ruiz, J., Lycan, T. W., Topaloglu, U., Chou, P. C., Miller, L. D., Hawkins, G. A., Alexander-Miller, M. A., O'Neill, S. S., Powell, B. L., D'Agostino, R. B., Jr, Munden, R. F., Pasche, B., \& Zhang, W. (2021). SMARCA4 mutations in KRAS-mutant lung adenocarcinoma: a multicohort analysis. Molecular oncology, 15(2), 462-472. doi:10.1002/1878-0261.12831

\section{Tables}


Table 1. Clinical Characteristics of 47 patients with BRG1-Deficient Lung Cancers.

\begin{tabular}{|c|c|}
\hline Parameter & Number(\%) \\
\hline \multicolumn{2}{|l|}{ Age at diagnosis $(y)(n=47)$} \\
\hline Median & 63 \\
\hline Range & $37-83$ \\
\hline \multicolumn{2}{|l|}{$\operatorname{Sex}(n=47)$} \\
\hline Male & $42(89.4)$ \\
\hline Female & $5(10.6)$ \\
\hline \multicolumn{2}{|l|}{ Smoking history $(n=47)$} \\
\hline Former or Current & $36(76.4)$ \\
\hline Never & $11(23.4)$ \\
\hline \multicolumn{2}{|l|}{ Stage at diagnosis $(n=47)$} \\
\hline $\mid-I I$ & $10(21.3)$ \\
\hline III-IV & $37(78.7)$ \\
\hline \multicolumn{2}{|l|}{ Distant metastases at diagnosis $(n=47)$} \\
\hline Present & $21(44.7)$ \\
\hline Absent & $26(55.3)$ \\
\hline \multicolumn{2}{|l|}{ Surgical resection( $\mathrm{N}=18)$} \\
\hline Adjuvant chemotherapy & $9(50)$ \\
\hline Recurrence & $5(27.8)$ \\
\hline Absent & $13(72.2)$ \\
\hline \multicolumn{2}{|l|}{ Advanced stage $(\mathrm{N}=29)$} \\
\hline Chemotherapy & 11(37.9) \\
\hline ICI(include combination) & $8(27.6)$ \\
\hline Chemotherapy combined other therapies & $4(13.8)$ \\
\hline Osimertinib & $1(3.45)$ \\
\hline Gamma Knife & $1(3.45)$ \\
\hline Palliative & $2(6.9)$ \\
\hline Not avaliable & $2(6.9)$ \\
\hline \multicolumn{2}{|l|}{ Follow-up time.mo(n=47) } \\
\hline Mean & 14 \\
\hline Range & $1-68$ \\
\hline \multicolumn{2}{|l|}{ Prognosis(n=47) } \\
\hline Disease free & $13(27.7)$ \\
\hline Survival with tumor & $5(10.6)$ \\
\hline Death & $29(61.7)$ \\
\hline \multicolumn{2}{|l|}{ Median OS.mo(n=47) } \\
\hline Surgical resection $(\mathrm{N}=18)$ & Not reached \\
\hline Advanced stage $(\mathrm{N}=29)$ & 7.0 \\
\hline
\end{tabular}


Table 2. ICls-based immunotherapy response for BRG1-deficient non-small cell lung cancers.

\begin{tabular}{lllllll} 
No & PD-L1 level & PD-1/PD-L1 inhibitor & Combined therapy & PFS(m) & OS(m) & Prognosis \\
\hline 19 & $95 \%$ & Nivolumab(First-line) & None & $37^{*}$ & $37^{*}$ & Alive \\
\hline 20 & $1 \%$ & Sintilimab(Second-line) & None & 5 & 15 & Dead \\
\hline 21 & $0 \%$ & Pembrolizumab(Second-line) & Docetaxel & $20^{*}$ & $22^{*}$ & Alive \\
\hline 30 & $3 \%$ & Sintilimab(Second-line) & None & $14^{*}$ & $26^{*}$ & Alive \\
\hline 35 & NR & Pembrolizumab(Third-line) & Anlotinib & $31^{*}$ & $39^{*}$ & Alive \\
\hline 37 & NR & Nivolumab(First-line) & TCb+RT & 3 & 3 & Dead \\
\hline 43 & $75 \%$ & Sintilimab(First-line) & Pemetrexed & 2 & 4 & Dead \\
\hline 44 & $25 \%$ & Nivolumab(First-line) & CP+RT & 14 & 14 & Dead
\end{tabular}

Abbreviations: NR, unavaliable; CP, Carboplatin+Pemetrexed; RT, radiotherapy; TCb, Docetaxel+Carboplatin;

*, Not reached.

Table 3-1. Pathological and Immunohistichemical features of surgical patients with BRG1-deficient non-small cell lung cancer.

\begin{tabular}{|c|c|c|c|c|c|c|c|c|c|c|c|c|c|}
\hline No & Histology & BRG1 & BRM & ARID1B & ARID1A & PD-L1 & TTF- & NapsinA & P40 & CK7 & $\begin{array}{l}\text { HepPar- } \\
1\end{array}$ & $\begin{array}{l}\text { ki- } \\
67\end{array}$ & EGFR/KRAS \\
\hline 1 & Solid ADCA & Loss & Loss & Intact & Intact & - & - & - & - & + & + & $60 \%$ & NR \\
\hline 2 & NKSCC & Loss & Intact & Intact & Intact & - & - & - & + & + & - & $70 \%$ & NR \\
\hline 3 & Solid ADCA & Loss & Intact & Intact & Intact & High(100\%) & + & + & - & + & - & $80 \%$ & NR \\
\hline 4 & Solid ADCA & Loss & Intact & Intact & Intact & - & - & - & - & + & - & $80 \%$ & NR \\
\hline 5 & Mucinous ADCA & Loss & Intact & Intact & Intact & - & - & - & - & + & + & $70 \%$ & - \\
\hline 6 & Solid ADCA & Loss & Intact & Intact & Intact & High(100\%) & - & - & - & + & - & $70 \%$ & NR \\
\hline 7 & Solid ADCA & Loss & Loss & Intact & Intact & - & + & $20 \%$ & - & + & $+\bigotimes 50 \%$ & $50 \%$ & NR \\
\hline 8 & Solid ADCA & Loss & Loss & Intact & Intact & - & + & + & - & + & - & $50 \%$ & NR \\
\hline 9 & Solid ADCA & Loss & Intact & Intact & Intact & - & - & - & - & - & + & $60 \%$ & NR \\
\hline 10 & Solid ADCA & Loss & Intact & Intact & Intact & $\operatorname{Low}(2 \%)$ & - & - & - & + & $+\bigotimes 10 \%$ & $60 \%$ & - \\
\hline 11 & $\begin{array}{l}\text { Solid ADCA } \\
\text { \acinar+micropapillary } \rrbracket\end{array}$ & Loss & Intact & Intact & Intact & - & + & $50 \%$ & - & + & - & $80 \%$ & NR \\
\hline 12 & Solid ADCA & Loss & SR & Intact & Intact & $\operatorname{Low}(5 \%)$ & - & - & - & + & $+\bigotimes 50 \%$ & $70 \%$ & NR \\
\hline 13 & Pleomorphic & Loss & SR & Intact & Intact & $\operatorname{High}(80 \%)$ & - & - & $\begin{array}{l}+\bigotimes \\
20 \%\end{array}$ & + & - & $80 \%$ & NR \\
\hline 14 & Solid ADCA+acinar & $\mathrm{PL}$ & Intact & Intact & Intact & - & $+\llbracket 5 \%$ & $\Delta \otimes 5 \%$ & - & + & + & $30 \%$ & - \\
\hline 15 & $\begin{array}{l}\text { Solid ADCA } \\
\text { 『Acinarmicropapillary } \rrbracket\end{array}$ & $\mathrm{PL}$ & Intact & Intact & Intact & $\operatorname{Low}(2 \%)$ & $+\llbracket 5 \%$ & $+\square 5 \%$ & - & + & $+\bigotimes 50 \%$ & $50 \%$ & NR \\
\hline 16 & Solid ADCA & $\mathrm{PL}$ & $\mathrm{PL}$ & Intact & Intact & - & $\begin{array}{l}+ \\
\nabla W K \nabla\end{array}$ & - & - & + & - & $70 \%$ & NR \\
\hline 17 & Solid ADCA & $\mathrm{PL}$ & Intact & Intact & Intact & High(80\%) & + & - & - & + & - & $80 \%$ & NR \\
\hline 18 & Solid ADCA & SR & SR & Intact & Intact & - & - & - & - & + & + & $70 \%$ & NR \\
\hline
\end{tabular}

Abbreviations: ADCA,adenocarcinoma; NSCLC,NOS, non-small cell lung cancer, not otherwise specified; NR, unavailable; SR, significantly reduced but not a completely loss; WK,weak; PL, partially loss.

Table 3-2. Pathological and Immunohistichemical features of advanced patients with BRG1-deficient non-small cell lung cancer. 


\begin{tabular}{|c|c|c|c|c|c|c|c|c|c|c|c|c|c|}
\hline No & Histology & BRG1 & BRM & ARID1A & ARID1B & PD-L1 & $\begin{array}{l}\text { TTF- } \\
1\end{array}$ & NapsinA & P40 & CK7 & $\underset{1}{\mathrm{HepPar}-}$ & $\begin{array}{l}\text { ki- } \\
67\end{array}$ & $\begin{array}{l}\text { EGFR/KRAS// } \\
1\end{array}$ \\
\hline 19 & Solid ADCA & Loss & Intact & Intact & Intact & $\operatorname{High}(95 \%)$ & - & - & - & + & - & $70 \%$ & - \\
\hline 20 & Adenosquamous & Loss & NR & NR & NR & $\operatorname{Low}(1 \%)$ & - & - &,$+ 20 \%$ & + & NR & $60 \%$ & - \\
\hline 21 & Solid ADCA & Loss & Loss & Intact & Intact & - & $\begin{array}{l}+\square \\
40 \%\end{array}$ & - & - & - & - & $70 \%$ & - \\
\hline 22 & Solid ADCA & Loss & Intact & Intact & Intact & - & + & - & - & + & - & $40 \%$ & - \\
\hline 23 & Solid ADCA & Loss & Intact & Intact & Intact & $\operatorname{Low}(5 \%)$ & - & - & - & + & $+\square 50 \%$ & $30 \%$ & - \\
\hline 24 & Solid ADCA & Loss & SR & Intact & Intact & $\operatorname{High}(50 \%)$ & - & - & - & + & - & $60 \%$ & - \\
\hline 25 & Solid ADCA & Loss & NR & Intact & Intact & - & - & - & - & + & + & $40 \%$ & NR \\
\hline 26 & Solid ADCA & Loss & NR & NR & NR & - & + & + & - & + & NR & $70 \%$ & EGFR exon 19 \\
\hline 27 & Solid ADCA & Loss & Intact & Intact & Intact & - & - & - & - & + & + & $90 \%$ & - \\
\hline 28 & NSCLC, NOS(PDCA) & Loss & NR & Intact & Intact & - & - & - & - & + & - & $50 \%$ & - \\
\hline 29 & Solid ADCA & Loss & SR & Intact & Intact & $\operatorname{Low}(20 \%)$ & - & - & - & $\begin{array}{l}+\square \\
10 \%\end{array}$ & - & $90 \%$ & - \\
\hline 30 & Solid ADCA & Loss & NR & NR & NR & $\operatorname{Low}(3 \%)$ & - & - & - & + & + & $80 \%$ & NR \\
\hline 31 & Acinar ADCA & Loss & NR & NR & NR & NR & - & NR & - & + & NR & $20 \%$ & NR \\
\hline 32 & Solid ADCA & Loss & SR & Intact & Intact & - & - & - & - & + & $+\llbracket 50 \%$ & $70 \%$ & NR \\
\hline 33 & Solid ADCA & Loss & Intact & Intact & Intact & - & - & - & - & + & - & $60 \%$ & NR \\
\hline 34 & Papillary+solid+acinar & Loss & Loss & Intact & Intact & - & - & $\mathbb{8} 30 \%$ & - & + & - & $90 \%$ & - \\
\hline 35 & NSCLC, NOS(PDCA) & Loss & NR & $N R$ & NR & NR & - & - & - & + & + & $40 \%$ & - \\
\hline 36 & Solid ADCA & Loss & Intact & Intact & Intact & $\operatorname{High}(50 \%)$ & - & - & - & + & - & $30 \%$ & - \\
\hline 37 & Solid ADCA & Loss & NR & NR & NR & NR & $\begin{array}{l}+\square \\
5 \%\end{array}$ & - & - & + & + & $90 \%$ & - \\
\hline 38 & Solid ADCA & Loss & SR & Intact & Intact & - & - & - & - & + & - & $30 \%$ & - \\
\hline 39 & Solid ADCA+acinar & Loss & NR & Intact & Intact & $\operatorname{Low}(5 \%)$ & - & - & - & + & + & $50 \%$ & KRAS exon2 \\
\hline 40 & Solid ADCA & Loss & NR & NR & NR & $\operatorname{Low}(1 \%)$ & - & - & - & - & - & $30 \%$ & NR \\
\hline 41 & Solid ADCA & Loss & Loss & Intact & Intact & - & - & - & - & + & - & $60 \%$ & - \\
\hline 42 & Solid ADCA+acinar & PL & Loss & Intact & Intact & - & $\mathbb{8} \nabla \%$ & $\mathbb{\Delta} \triangle 5 \%$ & - & + & - & $40 \%$ & KRAS exon2 \\
\hline 43 & Acinar ADCA & $\mathrm{PL}$ & NR & NR & NR & $\operatorname{High}(70 \%)$ & $\begin{array}{l}+\square \\
50 \%\end{array}$ & - & - & + & NR & $70 \%$ & KRAS exon2 \\
\hline 44 & Solid ADCA+acinar & SR & NR & NR & NR & $\operatorname{Low}(5 \%)$ & $10 \%$ & $+\rrbracket 10 \%$ & - & + & + & $25 \%$ & NR \\
\hline 45 & Solid ADCA & SR & NR & NR & NR & $\operatorname{Low}(5 \%)$ & - & - & - & + & + & $50 \%$ & KRAS exon2 \\
\hline 46 & Solid ADCA & $\mathrm{SR}$ & Intact & Intact & Intact & High(95\%) & - & - & - & + & - & $70 \%$ & EGFR exon20 \\
\hline 47 & Solid ADCA+acinar & SR & Intact & Intact & Intact & - & - & - & - & + & + & $70 \%$ & KRAS exon2 \\
\hline
\end{tabular}

Abbreviations: ADCA,adenocarcinoma; NKSCC,non-keratinizing squamous cell carcinoma; NR, unavailable; SR, significantly reduced but not a completely loss; WK,weak; PL, partially loss.

\section{Figures}



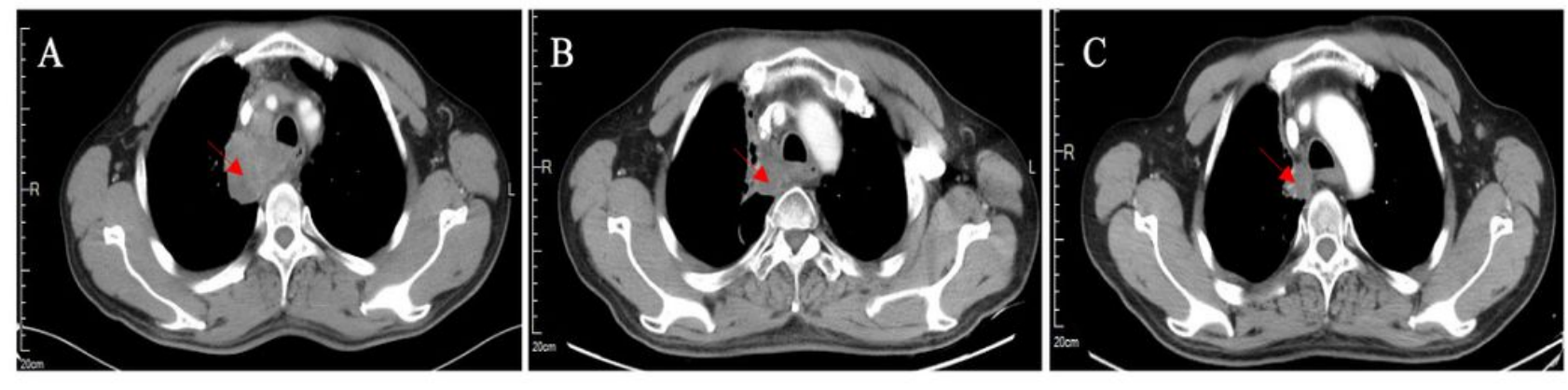

Figure 1

CT images of one patient received pembrolizumab combined anlotinib as third-line treatment. Arrows indicate tumors. (A) Pretreatment computed tomography(CT) scan of the patient; (B) CT scan after three cycles of pembrolizumab combined anlotinib; (C) CT scan after twenty-one cycles of pembrolizumab combined anlotinib.
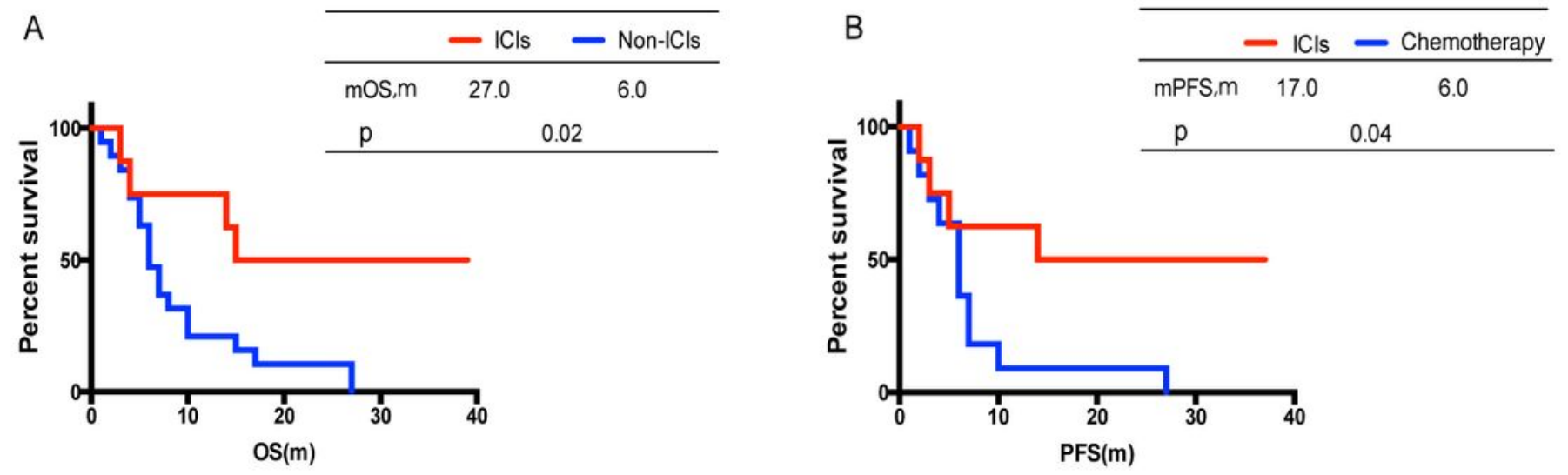

Figure 2

The efficacy of ICls-based immunotherapy in BRG1-deficient NSCLCs. (A) Over survival(OS) on ICls treated and non-ICls treated patients. Kaplan-Meier curve showing better median OS on ICls treated group $(\mathrm{p}=0.02)$. (B)Progression-free survival(PFS) on ICls treated and platinum doublet chemotherapy treated patients. Kaplan-Meier curve showing better median PFS on ICls treated group $(p=0.04)$. 

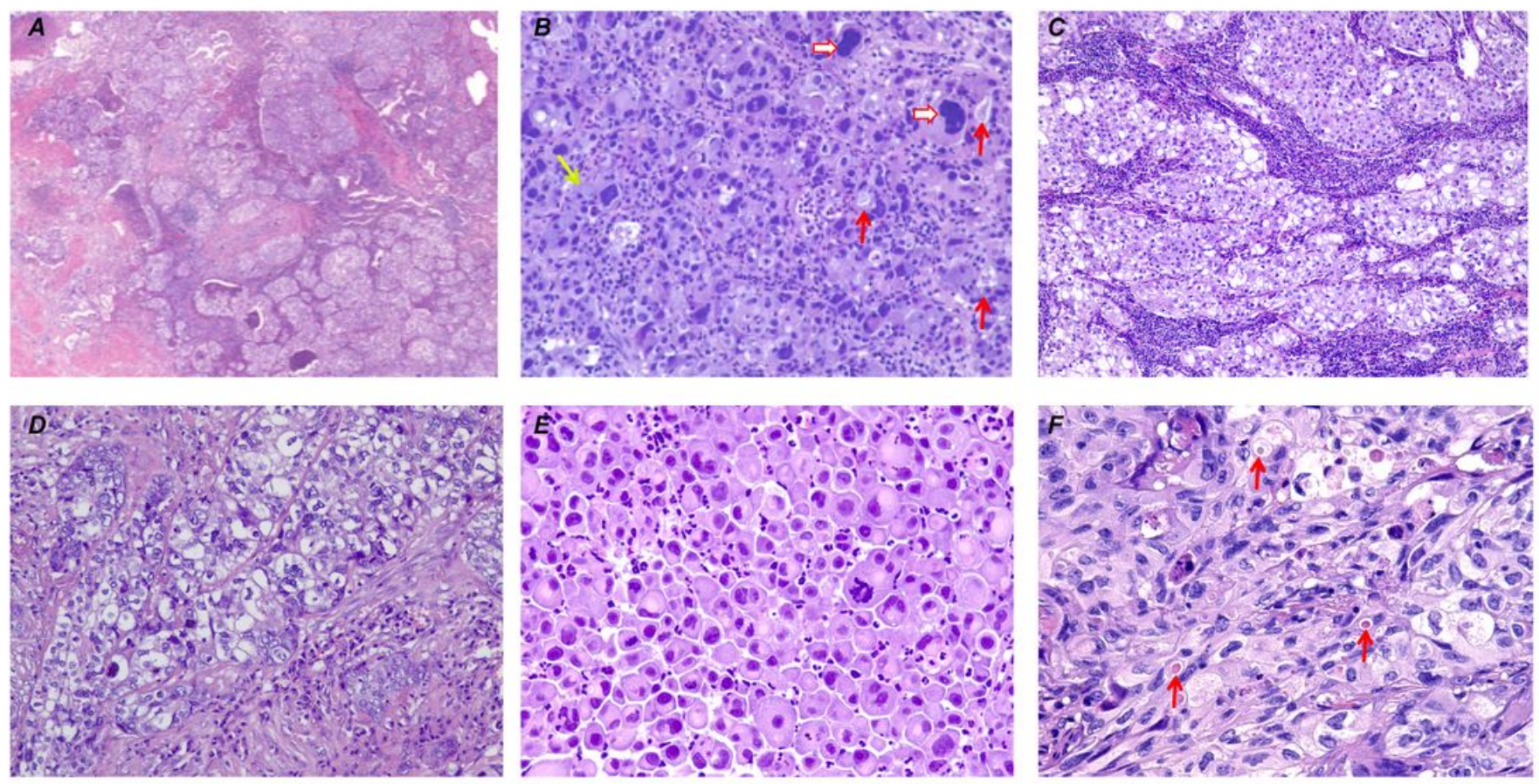

Figure 3

Morphologic features of BRG1-deficient NSCLCs. (A) Pure poorly differentiated solid adenocarcinoma with necrosis; (B) Abortive small lumina (red solid arrows),scattered goblet-like cells (yellow solid arrows) and pleomorphic giant cells and bizzare cells(hollow arrows) with intraepithelial infiltrate of inflammatory cells; (C) Large polygonal tumour cells arranged in compact solid nests and sheets with prominent stromal inflammation; (D) Tumor cells showed clear cytoplasm and vesicular chromatin; (E) Rhabdoid tumor cells with distinct eosinophilic nucleoli and a high mitotic rate; (F) Cytoplasmic hyaline secretory globules(single arrows) (hematoxylin-eosin, original magnification $\times 50$ [A]; original magnifications $\times 200[\mathrm{~B}$ and $\mathrm{D}]$; original magnifications $\times 100[\mathrm{C}]$ and $\times 400[E$ and $F]$ ). 

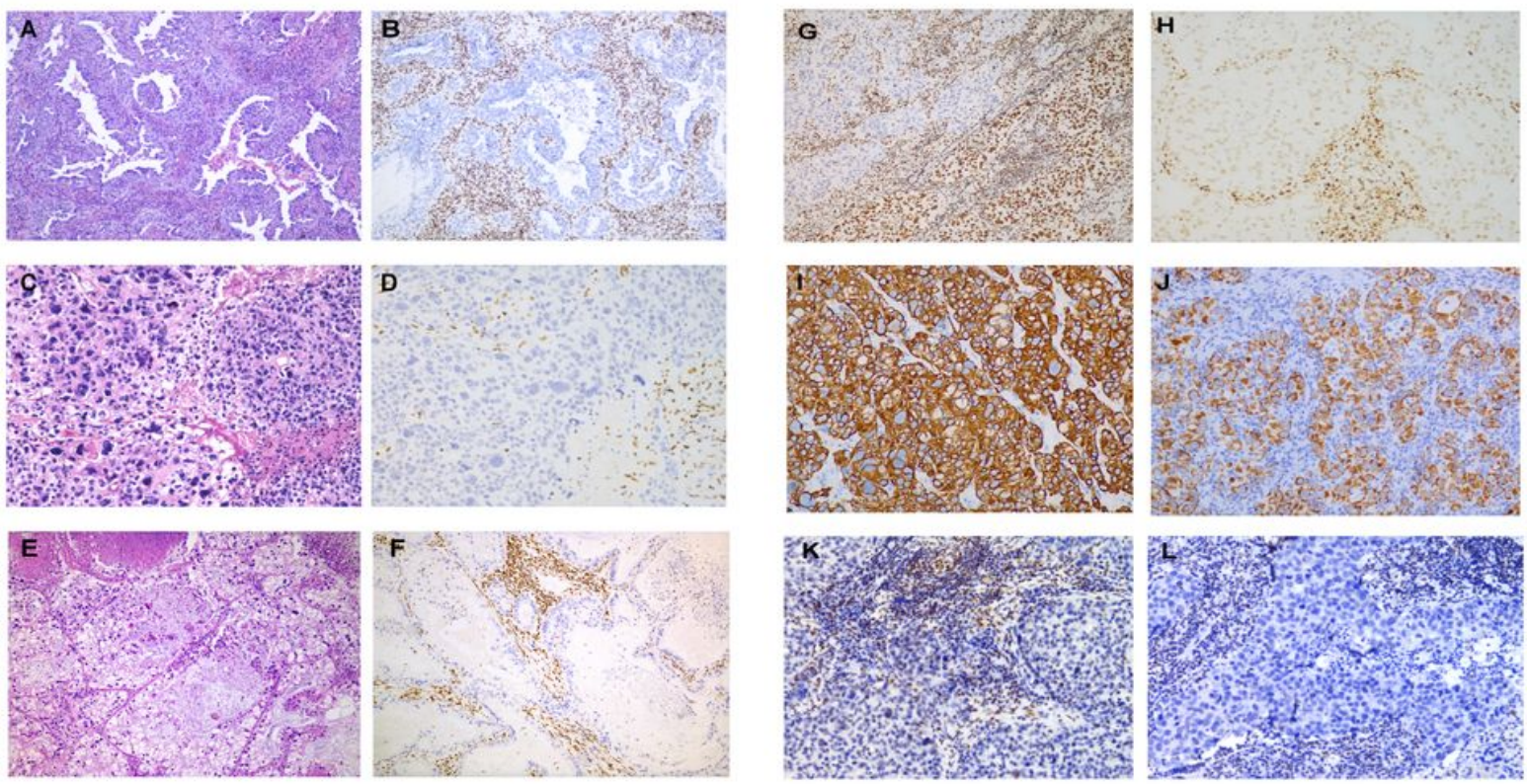

\section{Figure 4}

Morphologic and immunohistochemical features of BRG1-deficient NSCLCs. (A-B) Papillary pattern adenocarcinoma with complete loss of BRG1; (C-D) Pleomorphic carcinoma with complete loss of BRG1; (E-F) Acinar adenocarcinoma showed clear cytoplasm and necrosis with complete loss of BRG1; (G) Tumors showed partial loss of BRG1:BRG1 was expressed in the neoplastic cells on the right lower area and absent on the left upper area; (H) BRG1 expression was significantly reduced in contrast to strong staining in stromal cells; (I) Diffuse strong expression of CK7; ( $)$ Strong granular cytoplasmic expression of HepPar-1; (K-L)Tumors showed complete loss of BRM or significantly reduced of BRM (hematoxylin-eosin, original magnification×100 [A,B,E,F,G and $\mathrm{H}$,$] and \times 200[\mathrm{C}, \mathrm{D}, \mathrm{I}, \mathrm{J}, \mathrm{K}$ and $\mathrm{L}])$.

\section{Supplementary Files}

This is a list of supplementary files associated with this preprint. Click to download.

- sup.table.1antibodyinformation.docx 